\title{
A RECURRENCE FORMULA FOR $q$-BERNOULLI NUMBERS ATTACHED TO FORMAL GROUP
}

\author{
JUNYA SATOH
}

\begin{abstract}
Kaneko [2] proved a new recurrence formula for the Bernoulli numbers and gave two proofs. One of them was due to Don Zagier. We shall apply Zagier's idea to the $q$-Bernoulli numbers attached to formal group.
\end{abstract}

\section{Generalization of Kaneko's recurrence formula}

Let $\mathfrak{B}=\mathfrak{B}(X)$ be the generating function of the Bernoulli numbers, i.e.,

$$
\mathfrak{B}=\frac{X}{e^{X}-1},
$$

then it is anti-invariant under a map: $\mathfrak{B} \mapsto \mathfrak{B} e^{X}$, i.e.,

$$
\mathfrak{B}(X) e^{X}=\mathfrak{B}(-X) .
$$

Zagier gave another proof of Kaneko's recurrence formula for the Bernoulli numbers by using this property [2]. On the other hand because of $\mathfrak{B}(-X)=$ $\mathfrak{B}(X)+X$, we can see that $\mathfrak{B}$ is transformed to the sum of a polynomial and itself under the above map. We use the second property in order to generalize Kaneko's recurrence formula and prove a formula for the $q$-Bernoulli numbers attached to formal group.

First we suppose a power series $B$ in $X$ which satisfies the following:

\section{Assumption 1.}

$$
B e^{X}=B+C,
$$

where $C$ is a polynomial.

If $C=X$, then $B$ is equal to $\mathfrak{B}$, and if $C=X^{2}$, then $B$ is essentially equal to the generating function of $\tilde{B}_{n}$ which was defined in [2], i.e.,

$$
\sum_{n \geq 0} \tilde{B}_{n} \frac{X^{n}}{n !}=\left(\frac{X^{2}}{e^{X}-1}\right)^{\prime} .
$$

Received November 4, 1998. 
The starting point of our argument is the following trivial lemma:

LEMMA 1. For any power series $A$ and non-negative integer $n$, we have

$$
A^{(n)} e^{X}=\sum_{i=0}^{n}\left(\begin{array}{c}
n \\
i
\end{array}\right)(-1)^{n-i}\left(A e^{X}\right)^{(i)}
$$

where $A^{(n)}$ means the $n$-th derivative of $A$.

Proof. Because of $A=\left(A e^{X}\right) e^{-X}$, we can get what we want.

Set $A=B$ and compare the coefficient of $\frac{X^{m}}{m !}$ for any non-negative integer $m$, then we have a generalization of Kaneko's recurrence formula as follows:

Proposition 1. If $B$ satisfies Assumption 1, then we have

$$
\sum_{i=0}^{m}\left(\begin{array}{c}
m \\
i
\end{array}\right) b_{n+i}=\sum_{i=0}^{n}\left(\begin{array}{c}
n \\
i
\end{array}\right)(-1)^{n-i}\left(b_{m+i}+c_{m+i}\right),
$$

where $B=\sum_{n \geq 0} b_{n} \frac{X^{n}}{n !}$ and $C=\sum_{n \geq 0} c_{n} \frac{X^{n}}{n !}$.

If $m>\operatorname{deg} C$, then we have

Corollary 1.

$$
\sum_{i=0}^{m}\left(\begin{array}{c}
m \\
i
\end{array}\right) b_{n+i}=\sum_{i=0}^{n}\left(\begin{array}{c}
n \\
i
\end{array}\right)(-1)^{n-i} b_{m+i}
$$

Furthermore if $m=n$, then we have

Corollary 2.

$$
\sum_{\substack{i=0 \\
i \neq n \bmod 2}}^{n}\left(\begin{array}{c}
n \\
i
\end{array}\right) b_{n+i}=0
$$

If $C=X$, then there is no new information about the Bernoulli numbers. But if $C=X^{2}$, then this is equivalent to Kaneko's recurrence formula. 


\section{2. $q$-recurrence formula}

In this section we shall extend results in the previous section for the $q$-Bernoulli numbers attached to formal group. Let $q$ be an indeterminate and let $\mathfrak{o}$ be the formal power series ring in $q-1$ over some $\mathbb{Q}$-algebra. Furthermore let $\mathfrak{F}(X, Y)$ be a 1-dimensional commutative formal group defined over $\mathfrak{o}$ and let $\mathfrak{f}(X)$ be an isomorphism from the additive formal group $X+Y$ to $\mathfrak{F}(X, Y)$. We note that there exists a unique isomorphism $\mathfrak{f}_{\mathfrak{F}}(X)$ from $X+Y$ to $\mathfrak{F}(X, Y)$ defined over o such that $\mathfrak{f}_{\mathfrak{F}}^{\prime}(0)=1$. And $\mathfrak{f}(X)$ is equal to $\mathfrak{f}_{\mathfrak{F}}(c X)$ for some invertible element $c \in \mathfrak{o}^{\times}$. Conversely for any $c \in \mathfrak{o}^{\times}$, $\mathfrak{f}_{\mathfrak{F}}(c X)$ is an isomorphism from $X+Y$ to $\mathfrak{F}(X, Y)$. Throughout this paper we assume that

\section{Assumption 2.}

$$
\operatorname{ord}_{q-1} \mathfrak{f}_{\mathfrak{F}}^{(n)}(0) \geq n-1 \quad \text { for all } n \geq 1
$$

We note that by this assumption $\mathfrak{F}_{n}(A, B)$ (see Definition 1 below) and $\mathfrak{f}(a)$ are convergent in $\mathfrak{o}$ for any $A, B \in \mathfrak{o}[[X]]$ and $a \in \mathfrak{o}$ as formal power series (see [6, Remark 3]).

Definition 1. For each non-negative integer $n$, we denote the expansion of $\mathfrak{F}(X, Y)^{n}$ by

$$
\mathfrak{F}(X, Y)^{n}=\sum_{i, j \geq 0}\left(\begin{array}{c}
n \\
i, j
\end{array}\right)_{\mathfrak{F}} X^{i} Y^{j}
$$

and we set

$$
\mathfrak{F}_{n}(A, B)=\sum_{i, j \geq 0}\left(\begin{array}{c}
n \\
i, j
\end{array}\right)_{\mathfrak{F}} a_{i} b_{j}
$$

for any power series $A=\sum_{n \geq 0} a_{n} \frac{X^{n}}{n !}$ and $B=\sum_{n \geq 0} b_{n} \frac{X^{n}}{n !}$ in $\mathfrak{o}[[X]]$. Then we define the $* \mathfrak{F}$-product by

$$
A *_{\mathfrak{F}} B=\sum_{n \geq 0} \mathfrak{F}_{n}(A, B) \frac{X^{n}}{n !} .
$$

We can prove $\left(\mathfrak{o}[[X]],+, *_{\mathfrak{F}}\right)$ is an o-algebra (see $[6$, Proposition 1$\left.]\right)$. Next we extend the following map:

$$
X^{n} \mapsto c^{n} \underbrace{X *_{\mathfrak{F}} \cdots *_{\mathfrak{F}} X}_{n \text { times }}
$$


$\mathfrak{o}$-linearly. Hence we can get a natural homomorphism from $(\mathfrak{o}[[X]],+, \cdot)$ to $\left(\mathfrak{o}[[X]],+, *_{\mathfrak{F}}\right)$. We call this map $q$-operator and denote the image of $A \in \mathfrak{o}[[X]]$ under the $q$-operator by $A_{\mathfrak{F}, c}$. We define a $q$-analogue of power series $A$ attached to $\mathfrak{F}$ and $c$ by $A_{\mathfrak{F}, c}$. The following proposition is essential for our theory of $q$-analogue (see [6, Theorem 1 and Proposition 2]).

Proposition 2. For any $a, b \in \mathfrak{o}$, we have

(i) $\left(e^{a X}\right)_{\mathfrak{F}, c}=e^{\mathfrak{f}(a) X}$,

(ii) $e^{\mathfrak{f}(a) X} *_{\mathfrak{F}} e^{\mathfrak{f}(b) X}=e^{\mathfrak{f}(a+b) X}$.

We define the $q$-Bernoulli numbers $\beta_{n}(\mathfrak{F}, c)$ attached to $\mathfrak{F}$ and $c$ as follows:

Definition 2. For each non-negative integer $n$, we define the $n$-th $q$ Bernoulli number $\beta_{n}(\mathfrak{F}, c)$ attached to $\mathfrak{F}(X, Y) \in \mathfrak{o}[[X, Y]]$ and $c \in \mathfrak{o}^{\times}$by the coefficient of $\frac{X^{n}}{n !}$ in $\mathfrak{B}_{\mathfrak{F}, c}=\left(\frac{X}{e^{X}-1}\right)_{\mathfrak{F}, c}$.

We note that if $\mathfrak{F}=X+Y+(q-1) X Y$ and $c=\frac{\log q}{q-1}$, then $\mathfrak{f}(X)=\frac{q^{X}-1}{q-1}$ and $\beta_{n}(\mathfrak{F}, c)$ satisfies the following recurrence formula:

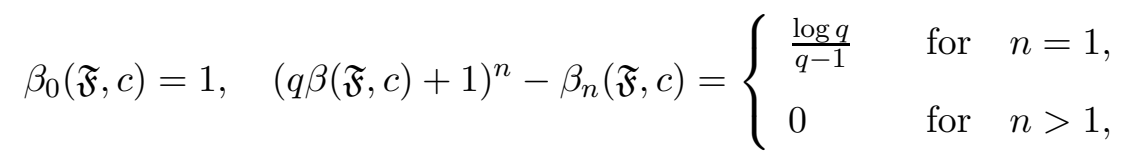

where we use the usual convention about replacing $\beta(\mathfrak{F}, c)^{i}$ by $\beta_{i}(\mathfrak{F}, c)$ for each non-negative integer $i$.

Proof. Apply the $q$-operator to $\mathfrak{B} e^{X}-\mathfrak{B}=X$, then we have $\mathfrak{B}_{\mathfrak{F}, c} *_{\mathfrak{F}}$ $e^{X}-\mathfrak{B}_{\mathfrak{F}, c}=c X$ and $\mathfrak{F}_{n}\left(\mathfrak{B}_{\mathfrak{F}, c}, e^{X}\right)=(q \beta(\mathfrak{F}, c)+1)^{n}$. Hence the above recurrence formula holds.

Now we may get a $q$-analogue of Proposition 1 by applying the $q$ operator to Lemma 1 , but it is unknown the commutativity of the $q$-operator and the derivative on $\mathfrak{o}[[X]]$. So we need to take another approach to get a $q$-analogue of Lemma 1. 
LEMma 2. For any power series $A, B$ and non-negative integer $n$, we have

$$
\left(A *_{\mathfrak{F}} B\right)^{(n)}=\sum_{i, j \geq 0}\left(\begin{array}{c}
n \\
i, j
\end{array}\right)_{\mathfrak{F}} A^{(i)} *_{\mathfrak{F}} B^{(j)}
$$

Proof. For any non-negative integer $m$, the coefficient of $\frac{X^{m}}{m !}$ in the left hand side of (1) is equal to

$$
\mathfrak{F}_{m+n}(A, B)=\sum_{i, j \geq 0}\left(\begin{array}{c}
m+n \\
i, j
\end{array}\right)_{\mathfrak{F}} a_{i} b_{j}
$$

On the other hand that in the right hand of (1) is equal to

$$
\sum_{i, j \geq 0}\left(\begin{array}{c}
n \\
i, j
\end{array}\right)_{\mathfrak{F}} \mathfrak{F}\left(A^{(i)}, B^{(j)}\right)=\sum_{i, j \geq 0}\left(\begin{array}{c}
n \\
i, j
\end{array}\right)_{\mathfrak{F}} \sum_{k, l \geq 0}\left(\begin{array}{c}
m \\
k, l
\end{array}\right)_{\mathfrak{F}} a_{i+k} b_{j+l}
$$

Hence it is sufficient to show that

$$
\left(\begin{array}{c}
m+n \\
i, j
\end{array}\right)_{\mathfrak{F}}=\sum_{\substack{0 \leq k \leq i \\
0 \leq l \leq j}}\left(\begin{array}{c}
m \\
k, l
\end{array}\right)_{\mathfrak{F}}\left(\begin{array}{c}
n \\
i-k, j-l
\end{array}\right)_{\mathfrak{F}}
$$

for all $i \geq 0$ and $j \geq 0$. Because of $\mathfrak{F}(X, Y)^{m+n}=\mathfrak{F}(X, Y)^{m} \mathfrak{F}(X, Y)^{n}$, we can get what we want.

Apply this lemma to $A *_{\mathfrak{F}} e^{\mathfrak{f}(1) X}$ and $e^{\mathfrak{f}(-1) X}$, then we have

LEMMA 3.

$$
A^{(n)} *_{\mathfrak{F}} e^{\mathfrak{f}(1) X}=\sum_{i, j \geq 0}\left(\begin{array}{c}
n \\
i, j
\end{array}\right)_{\mathfrak{F}} \mathfrak{f}(-1)^{j}\left(A *_{\mathfrak{F}} e^{\mathfrak{f}(1) X}\right)^{(i)} .
$$

This is a $q$-analogue of Lemma 1. If $B$ satisfies Assumption 1, by applying the $q$-operator, we have

$$
B_{\mathfrak{F}, c} * \mathfrak{F} e^{\mathfrak{f}(1) X}=B_{\mathfrak{F}, c}+C_{\mathfrak{F}, c}
$$

If $C$ is a polynomial, then $C_{\mathfrak{F}, c}$ is also a polynomial and $\operatorname{deg} C=\operatorname{deg} C_{\mathfrak{F}, c}$ (see [6, Lemma 2]). Hence we have the following: 
Proposition 3. If $B$ satisfies Assumption 1, then we have

$$
\sum_{i, j \geq 0}\left(\begin{array}{c}
m \\
i, j
\end{array}\right)_{\mathfrak{F}} \mathfrak{f}(1)^{j} \beta_{n+i}=\sum_{i, j \geq 0}\left(\begin{array}{c}
n \\
i, j
\end{array}\right)_{\mathfrak{F}} \mathfrak{f}(-1)^{j}\left(\beta_{m+i}+\gamma_{m+i}\right)
$$

for any non-negative integer $m$ and $n$, where $B_{\mathfrak{F}, c}=\sum_{n \geq 0} \beta_{n} \frac{X^{n}}{n !}$ and $C_{\mathfrak{F}, c}=$ $\sum_{n \geq 0} \gamma_{n} \frac{X^{n}}{n !}$

If $m>\operatorname{deg} C$, then we have

Corollary 3.

$$
\sum_{i, j \geq 0}\left(\begin{array}{c}
m \\
i, j
\end{array}\right)_{\mathfrak{F}} \mathfrak{f}(1)^{j} \beta_{n+i}=\sum_{i, j \geq 0}\left(\begin{array}{c}
n \\
i, j
\end{array}\right)_{\mathfrak{F}} \mathfrak{f}(-1)^{j} \beta_{m+i}
$$

Furthermore if $m=n$, then we have

Corollary 4.

$$
\sum_{i, j \geq 0}\left(\begin{array}{c}
n \\
i, j
\end{array}\right)_{\mathfrak{F}}\left\{\mathfrak{f}(1)^{j}-\mathfrak{f}(-1)^{j}\right\} \beta_{n+i}=0
$$

Hence if $C=X$, then we get a recurrence formula for $\beta_{n}=\beta_{n}(\mathfrak{F}, c)$. On the other hand if $C=X^{2}$, then $\beta_{n}$ is the coefficient of $\frac{X^{n}}{n !}$ in

$$
\left(\frac{X^{2}}{e^{X}-1}\right)_{\mathfrak{F}, c}=c X *_{\mathfrak{F}} \mathfrak{B}_{\mathfrak{F}, c}=c X d_{\mathfrak{F}}\left(\mathfrak{B}_{\mathfrak{F}, c}\right),
$$

where $d_{\mathfrak{F}}=\sum_{i \geq 0}\left(\begin{array}{c}1 \\ i, 1\end{array}\right)_{\mathfrak{F}} \frac{d^{i}}{d X^{i}}$ (see $[6$, Lemma 1]). Furthermore if $\mathfrak{F}(X, Y)=$ $X+Y+(q-1) X Y$ and $c=\frac{\log q}{q-1}$, then $\frac{1}{c} d_{\mathfrak{F}}\left(\mathfrak{B}_{\mathfrak{F}, c}\right)$ is equal to the generating function of Carlitz's $q$-Bernoulli numbers (see the next section). This means that we get a Kaneko's type of recurrence formula for Carlitz's q-Bernoulli numbers.

3. $X+Y+(q-1) X Y$

If $\mathfrak{F}(X, Y)=X+Y+(q-1) X Y$ and $c=\frac{\log q}{q-1}$, then we can state results in the previous section as follows: 
Corollary 5. If $B$ satisfies Assumption 1, then we have

(i) $\sum_{i=0}^{m}\left(\begin{array}{c}m \\ i\end{array}\right) q^{n+i} \beta_{n+i}=\sum_{i=0}^{n}\left(\begin{array}{c}n \\ i\end{array}\right)(-1)^{n-i}\left(\beta_{m+i}+\gamma_{m+i}\right)$,

(ii) $\sum_{i=0}^{m}\left(\begin{array}{c}m \\ i\end{array}\right) q^{n+i} \beta_{n+i}=\sum_{i=0}^{n}\left(\begin{array}{c}n \\ i\end{array}\right)(-1)^{n-i} \beta_{m+i} \quad$ if $\quad m>\operatorname{deg} C$,

(iii) $\sum_{i=0}^{n}\left(\begin{array}{c}n \\ i\end{array}\right)\left\{q^{n+i}-(-1)^{n-i}\right\} \beta_{n+i}=0 \quad$ if $m=n>\operatorname{deg} C$,

where $B_{\mathfrak{F}, c}=\sum_{n \geq 0} \beta_{n} \frac{X^{n}}{n !}$ and $C_{\mathfrak{F}, c}=\sum_{n \geq 0} \gamma_{n} \frac{X^{n}}{n !}$.

Proof. If $\mathfrak{F}(X, Y)=X+Y+(q-1) X Y$, then, by the definition of $\left(\begin{array}{c}m \\ i, j\end{array}\right)_{\mathfrak{F}}$, we have

$$
\left(\begin{array}{c}
m \\
i, j
\end{array}\right)_{\mathfrak{F}}=\left(\begin{array}{c}
m \\
i
\end{array}\right)\left(\begin{array}{c}
i \\
i+j-m
\end{array}\right)(q-1)^{i+j-m} .
$$

Hence for any $a \in \mathfrak{o}$, we have

$$
\begin{aligned}
& \sum_{i, j \geq 0}\left(\begin{array}{c}
m \\
i, j
\end{array}\right)_{\mathfrak{F}} \mathfrak{f}(a)^{j} \beta_{n+i} \\
= & \sum_{i, j \geq 0}\left(\begin{array}{c}
m \\
i
\end{array}\right)\left(\begin{array}{c}
i \\
i+j-m
\end{array}\right)(q-1)^{i+j-m} \mathfrak{f}(a)^{j} \beta_{n+i} \\
= & \sum_{i=0}^{m}\left(\begin{array}{c}
m \\
i
\end{array}\right) \mathfrak{f}(a)^{n-i} \beta_{n+i} \sum_{j=m-i}^{m}\left(\begin{array}{c}
i \\
i+j-m
\end{array}\right)\{(q-1) \mathfrak{f}(a)\}^{i+j-m} \\
= & \sum_{i=0}^{m}\left(\begin{array}{c}
m \\
i
\end{array}\right) \mathfrak{f}(a)^{n-i} \beta_{n+i} q^{a i} .
\end{aligned}
$$

Hence we can get what we want.

Let $\bar{\beta}_{n}(\mathfrak{F}, c)$ be the coefficient of $\frac{X^{n}}{n !}$ in $\frac{1}{c} d_{\mathfrak{F}}\left(\mathfrak{B}_{\mathfrak{F}, c}\right)$, i.e., that of $c^{2} \frac{X^{n+1}}{n !}$ in $\left(\frac{X^{2}}{e^{X}-1}\right)_{\mathfrak{F}, c}$, then $\bar{\beta}_{n}(\mathfrak{F}, c)$ satisfies the following Carlitz's recurrence formula $([1])$ :

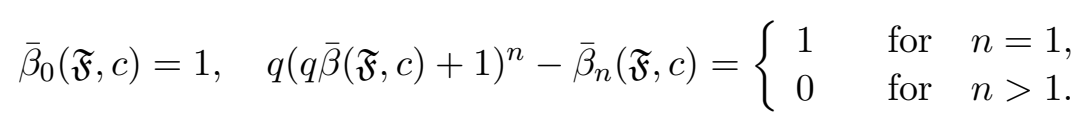


Hence $\bar{\beta}_{n}(\mathfrak{F}, c)$ is equal to the $n$-th Carlitz's $q$-Bernoulli number. To prove this we need the following:

LEMMA 4. If $\mathfrak{F}(X, Y)=X+Y+(q-1) X Y$, then $d_{\mathfrak{F}}$ is a homomorphism on $\left(\mathfrak{o}[[X]],+, *_{\mathfrak{F}}\right)$.

Proof. In this case we can write

$$
d_{\mathfrak{F}}(A)=A+(q-1) A^{\prime}
$$

for any power series $A \in \mathfrak{o}[[X]]$. Hence by Lemma 2 we have

$$
\begin{aligned}
d_{\mathfrak{F}}\left(A *_{\mathfrak{F}} B\right) & =A *_{\mathfrak{F}} B+(q-1)\left(A *_{\mathfrak{F}} B\right)^{\prime} \\
& =A *_{\mathfrak{F}} B+(q-1)\left\{A^{\prime} *_{\mathfrak{F}} B+A *_{\mathfrak{F}} B^{\prime}+(q-1) A^{\prime} *_{\mathfrak{F}} B^{\prime}\right\} \\
& =\left(A+(q-1) A^{\prime}\right) *_{\mathfrak{F}}\left(B+(q-1) B^{\prime}\right) \\
& =d_{\mathfrak{F}}(A) *_{\mathfrak{F}} d_{\mathfrak{F}}(B)
\end{aligned}
$$

for any $A$ and $B$ in $\mathfrak{o}[[X]]$. Hence $d_{\mathfrak{F}}$ is a homomorphism on $\left(\mathfrak{o}[[X]],+, *_{\mathfrak{F}}\right)$.

Proof (Carlitz's recurrence formula). Apply $d_{\mathfrak{F}}$ to $\mathfrak{B}_{\mathfrak{F}, c} *_{\mathfrak{F}} e^{X}-\mathfrak{B}_{\mathfrak{F}, c}=$ $c X$, then we have

$$
d_{\mathfrak{F}}\left(\mathfrak{B}_{\mathfrak{F}, c}\right) * \mathfrak{F} q e^{X}-d_{\mathfrak{F}}\left(\mathfrak{B}_{\mathfrak{F}, c}\right)=c X-\log q .
$$

Hence $\bar{\beta}_{n}(\mathfrak{F}, c)$ satisfies Carlitz's recurrence formula.

Finally we give another proof of Corollary 5.

LEMmA 5. If $\mathfrak{F}(X, Y)=X+Y+(q-1) X Y$, then the * $\mathfrak{F}_{\text {-product is }}$ written by

$$
A *_{\mathfrak{F}} X^{n}=d_{\mathfrak{F}}^{n}(A) X^{n}
$$

for any non-negative integer $n$.

Proof. It is sufficient to prove for $\frac{X^{i}}{i !} *_{\mathfrak{F}} \frac{X^{j}}{j !}(i \geq 0$ and $j \geq 0)$. By the definition of $*_{\mathfrak{F}}$ and (2), we have

$$
\begin{aligned}
\frac{X^{i}}{i !} * \mathfrak{F} \frac{X^{j}}{j !} & =\sum_{m \geq 0}\left(\begin{array}{c}
m \\
i, j
\end{array}\right) \frac{X^{m}}{m !} \\
& =\sum_{m=j}^{i+j}\left(\begin{array}{c}
m \\
i
\end{array}\right)\left(\begin{array}{c}
i \\
i+j-m
\end{array}\right)(q-1)^{i+j-m} \frac{X^{m}}{m !} .
\end{aligned}
$$


On the other hand

$$
\begin{aligned}
d_{\mathfrak{F}}^{i}\left(\frac{X^{j}}{j !}\right) \frac{X^{i}}{i !} & =\sum_{m=0}^{i}\left(\begin{array}{c}
i \\
m
\end{array}\right)(q-1)^{m} \frac{d^{m}}{d X^{m}}\left(\frac{X^{j}}{j !}\right) \frac{X^{i}}{i !} \\
& =\sum_{m=0}^{i}\left(\begin{array}{c}
i \\
m
\end{array}\right)\left(\begin{array}{c}
i+j-m \\
i
\end{array}\right)(q-1)^{m} \frac{X^{i+j-m}}{(i+j-m) !}
\end{aligned}
$$

Hence we have what we want.

Remark 1. If $\mathfrak{F}=X+Y+(q-1) X Y$ and $c=\frac{\log q}{q-1}$, then, by Lemma 5 , we can get

$$
A(X) *_{\mathfrak{F}} e^{\mathfrak{f}(a)}=A\left(q^{a} X\right) e^{\mathfrak{f}(a)}
$$

for any power series $A \in \mathfrak{o}[[X]]$ and $a \in \mathfrak{o}$. By this we can get Corollary 5 from Lemma 1 not using Lemma 2.

Remark 2. Lemma 4 and Lemma 5 hold only for $\mathfrak{F}(X, Y)=X+Y+$ $(q-1) X Y$ (see [6, Lemma 1 and Proposition 4]).

\section{REFERENCES}

[1] L. Carlitz, q-Bernoulli numbers and polynomials, J. Duke Math. J., 15 (1948), 987-1000.

[2] M. Kaneko, A recurrence formula for the Bernoulli numbers, Proc. Japan Acad., 71,Ser. A, No. 8 (1995), 192-193.

[3] J. Satoh, q-analogue of Riemann's $\zeta$-function and $q$-Euler numbers, J. Number Theory, 31 (1989), 346-362.

[4] — A construction of q-analogue of Dedekind sums, Nagoya Math. J., 127 (1992), 129-143.

[5] Construction of q-analogue by using Stirling numbers, Japan. J. Math., 20, No. 1 (1994), 73-91.

[6] - Another look at the q-analogue from the viewpoint of formal groups, Preprint Ser. in Math. Sciences, Nagoya Univ. (1999-4).

Graduate School of Human Informatics

Nagoya University

Furo-cho Chikusa-ku

Nagoya 464-8601, Japan

jsatoh@math.human.nagoya-u.ac.jp 